\title{
Comparison of three validated nutritional screening tools in the oncology setting
}

\author{
F. Roulston ${ }^{1}$ and R. McDermott ${ }^{2}$ \\ ${ }^{1}$ Department of Nutrition \& Dietetics and ${ }^{2}$ Department of Medical Oncology, Adelaide \& Meath Hospital, \\ Dublin 24, Republic of Ireland
}

Undernutrition is common among patients with cancer $^{(1)}$ resulting in a reduced quality of life, lower activity level, increased treatmentrelated adverse reactions, reduced tumour response to treatment and reduced survival ${ }^{(2)}$. Screening for undernutrition is therefore vital for all patients with cancer ${ }^{(3)}$.

The aim of the present study was twofold, i.e. to compare three validated nutritional screening tools (the mini nutritional assessment $(\mathrm{MNA})^{(4)}$, the malnutrition screening tool (MST) ${ }^{(5)}$ and the malnutrition universal screening tool (MUST) ${ }^{(6)}$ ) and to ascertain which tool is most appropriate in the oncology setting. For this purpose the dietetic records of fifty-two patients attending the oncology service were retrospectively reviewed. Each of the three screening tools were completed for each patient using data from their first nutritional assessment and then compared with the clinical judgement of the oncology dietitian following a full nutritional assessment.

Results showed that the MNA and the MST had a sensitivity index (percentage of total patients identified as being at risk of undernutrition by each of the screening tools) of 92 and 79 respectively, with the scores for the two tools being highly correlated. The MNA and the MST also showed a high correlation with the dietitian's clinical assessment. On the other hand, the MUST was less sensitive (sensitivity index 29), failing to identify thirty-two patients considered to be at risk of being undernourished. The Table details the levels of agreement between the various screening tools used in the study.

\begin{tabular}{|c|c|c|c|c|c|c|c|c|}
\hline \multirow[b]{2}{*}{ Screening tool } & \multicolumn{2}{|c|}{$\begin{array}{r}\text { MNA } \\
\end{array}$} & \multicolumn{2}{|c|}{ MST } & \multicolumn{2}{|c|}{ MUST } & \multicolumn{2}{|c|}{ Dietitian } \\
\hline & Score & $\%$ & Score & $\%$ & Score & $\%$ & Score & $\%$ \\
\hline MNA & & & 45 & 87 & 19 & 37 & 51 & \\
\hline MST & 45 & 87 & & & 24 & 46 & 46 & \\
\hline MUST & 19 & 37 & 24 & 46 & & & 20 & \\
\hline Dietitian & 51 & 98 & 46 & 88 & 20 & 38 & & \\
\hline
\end{tabular}

In conclusion, both the MNA and the MST are screening tools capable of correctly identifying oncology patients at risk of undernutrition. As step three of the MUST only allocates a score to patients who are acutely ill and have, or are likely to have, no nutritional intake for $>5 \mathrm{~d}$, it does not account for decreased nutritional intake or nutrition-related side effects from illness and/or treatment, which can often arise in patients with cancer. Thus, the MUST, although validated to be applied to all types of patient groups, does not appear to be appropriate in the oncology setting.

1. Mattox T (2005) Nutr Clin Pract 20, 400-410.

2. Arends J, Bodoky G, Bozzetti F et al. (2006) Clin Nutr 25, 245-259.

3. Huhmann M \& Cunningham R (2005) Lancet Oncol 6, 334-343.

4. Guigoz Y, Vellas B \& Garry PJ (1996) Nutr Rev 54, S59-S65.

5. Ferguson M, Capra S, Bauer J et al. (1999) Nutrition 15, 458-464.

6. Elia M (2003) Screening for Malnutrition: A Multidisciplinary Responsibility. Development and Use of the 'Malnutrition Universal Screening Tool' (MUST) for Adults. Redditch, Worcs.: BAPEN. 